\title{
Developing excellence in developmental psychiatry
}

\author{
C. M. Freitag' ${ }^{1}$ A. Reif $^{2}$
}

Published online: 19 July 2016

(c) Springer-Verlag Wien 2016

Mental disorders develop over the lifespan. As trivial as this statement may sound, as complicated are its implications. Disorders that are commonly considered to have their onset in adulthood often feature precursors in childhood or adolescence, e.g., depression can be preceded by anxiety disorders in infancy. In addition, we know by now that "typical" childhood disorders such as ADHD and autism persist into adulthood, but change clinical presentation considerably. Genetic factors have an important role in shaping this developmental trajectory and these may not only include classical common and rare genetic variants, that can easily be measured in bodily fluids, but also de novo germline and somatic mutations which might only be detectable in the brain. During brain development, which takes place until the 20s, a number of risk factors may occur: pre- and postnatal famine, viral infections, delivery complications, drug exposure, to name but a few. Beyond these biological mechanisms, of course also varying environmental influences affect the course of disease; traumatic life events, familial atmosphere and parenting style, socioeconomic stratum all may shape the progression of disorder (or, on the other hand, resilience against it). All of these factors (Fig. 1) interact-for instance, initial studies point to an

A. Reif

Andreas.Reif@kgu.de

C. M. Freitag

c.freitag@em.uni-frankfurt.de

1 Department of Child and Adolescent Psychiatry, Psychosomatic Medicine and Psychotherapy, University Hospital Frankfurt and German Center of Developmental Psychiatry, Frankfurt am Main, Germany

2 Department of Adult Psychiatry, Psychosomatic Medicine and Psychotherapy, University Hospital Frankfurt and German Center of Developmental Psychiatry, Frankfurt am Main, Germany interaction of risk gene variants with traumatic life events to increase the risk towards later-life depression and anxiety disorders. Importantly, all processes ultimately converge on the level of the brain where a dynamic landscape of neural function and structure is mirroring a highly adaptive and plastic genomic-transcriptomic network of the neuron.

Unsurprisingly, the apparent complexity mentioned above results in insufficient knowledge about the mechanisms that underlie mental disease trajectories. Costly longitudinal studies on an epidemiological level, including multi-level biological investigations, would be needed to address this issue, and still, only a small fraction of pathology could be picked up due to the inaccessibility of the target organ, the brain. Nevertheless, such studies are urgently needed to characterize the development of mental disorders along the lifespan on a granular level: only such attempts will provide data that can be used to identify predictive markers for early diagnosis and also preventive measures will benefit from this knowledge. According studies, however, call for a tight interaction between Child and Adolescent Psychiatry on the one side and Adult Psychiatry on the other side, which, however, only rarely is the case. While there are good reasons that both disciplines operate separately, there is a clear need for better collaboration especially regarding research efforts (and also clinical structures that target the transition period between the 16th and the 25th year of age) (Fig. 2).

To overcome this gap, we have founded the German Center of Developmental Psychiatry (D-ZEP) in Frankfurt. Also making use of the $\mathrm{rmn}^{2}$ framework (http://www.rmn2. de/), the D-ZEP comprises the Departments of Child, Adolescent and Adult Psychiatry, Psychosomatic Medicine and Psychotherapy of the University Hospitals Frankfurt and Mainz, the Departments of Clinical Psychology of both Universities, and the Departments of Biological and Medical 
Fig. 1 Different levels of risk factors influencing the developmental trajectory of mental disorders
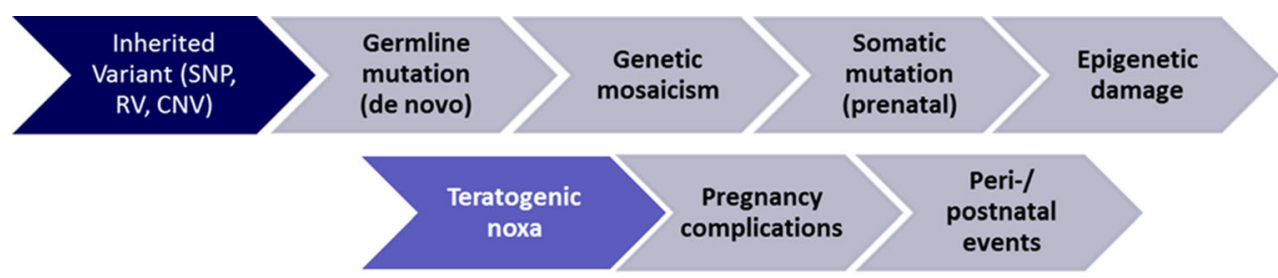

Chronic
psychosocial
risk factors $\quad \begin{gathered}\text { Early adverse } \\ \text { events }\end{gathered} \quad \begin{gathered}\text { Traumatic life } \\ \text { events during } \\ \text { adulthood }\end{gathered}$

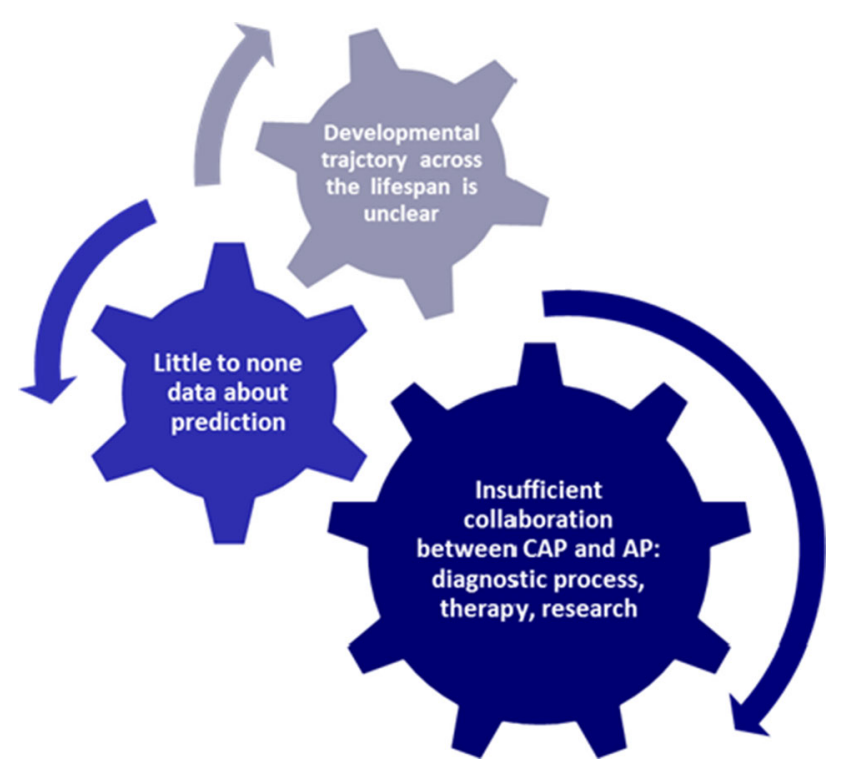

Fig. 2 Collaboration across disciplines is key to uncover the mechanisms underlying the trajectories of mental health

Psychology of the Goethe-University Frankfurt as well as the German Institute for International Educational Research. We feel that the local environment is ideally suited to host such a center, as there are already tight collaborations regarding according research questions, as especially evidenced by four large-scale projects funded by the EU: FemNAT-CD on female-specific aspects of conduct disorder (FP 7, coordinated by Freitag), Aggressotype on subtyping of aggression especially in ADHD and CD (FP 7, workpackage leader Reif), MiND on mechanisms underlying ADHD and autism (Horizon 2020, workpackage lead Reif) and CoCA on the comorbidities of ADHD (Horizon 2020, coordinated by Reif and workpackage lead by Freitag). The recently funded CRC 1193 on Neurobiological Mechanisms of Resilience underscores the potential of the Rhine-Main neuroscience community to tackle the complex issue how mental disorders develop over the lifespan. Our endeavor is supported by an excellent international scientific advisory board, which is in close contact with the D-ZEP PIs and continuously monitors our effort (for details, see list of members and scientific advisory board below).
This Special "Inaugural" Issue is to commemorate the foundation of the D-ZEP, which took place on the 21st of September 2015 in Frankfurt am Main. To do so, members of the D-ZEP and its advisory board were asked to provide a topical article which showcases the competence of the Center. We feel that our contributors superbly managed to do so. These articles bridge genetic, preclinical and clinical studies and hence display the full breadth of methods which are necessary to target our problems. We feel that these studies and reviews, in their entirety, are excellent examples how research can benefit from interdisciplinary collaboration and hope that our Center will continue to prosper in that vein!

\section{Founding Members of the German Center of Develop-} mental Psychiatry e.V. (D-ZEP):

A. Reif, Frankfurt am Main (Chair)

C. M. Freitag, Frankfurt am Main (Vice Chair)

J. Kaiser, Frankfurt am Main

U. Stangier, Frankfurt am Main

C. Fiebach, Frankfurt am Main

M. Hasselhorn, Frankfurt am Main

K. Lieb, Mainz

M. Huss, Mainz

M. Wessa, Mainz

We are very grateful for the support of our Scientific advisory board:

A. Heinz, Berlin

A. Meyer-Lindenberg, Mannheim

A. Fallgatter, Tübingen

M. Hautzinger, Tübingen

P. Pauli, Würzburg

S. Schneider, Bochm

T. Banaschewski, Mannheim

J. Hebebrand, Essen

B. Herpertz-Dahlmann, Aachen

S. Eliez, Geneva

J. Buitelaar, Nijmegen

S. Faraone, Syracuse

B. Franke, Nijmegen 